\title{
Dispositional and situational emotion regulation in younger and older adults
}

\author{
Hofer, Matthias ; Allemand, Mathias
}

\begin{abstract}
Younger and older adults $(\mathrm{N}=207)$ viewed a film scene eliciting strong negative emotions and then indicated how they had regulated their emotions. Dispositional emotion regulation was measured prior to stimulus presentation. Older adults showed higher levels of dispositional suppression than younger adults. Furthermore, individual differences in dispositional expressive suppression were predictive of reappraisal and suppression in the given situation. However, dispositional suppression was more strongly related to situational suppression among older adults than among younger adults. Future directions concerning the importance of and possible implications for age differences in coping with stressful situations are discussed.
\end{abstract}

DOI: https://doi.org/10.1024/1662-9647/a000171

Posted at the Zurich Open Repository and Archive, University of Zurich ZORA URL: https://doi.org/10.5167/uzh-145385

Journal Article

Accepted Version

Originally published at:

Hofer, Matthias; Allemand, Mathias (2017). Dispositional and situational emotion regulation in younger and older adults. GeroPsych, 30(3):109-118.

DOI: https://doi.org/10.1024/1662-9647/a000171 
Dispositional and Situational Emotion Regulation in Younger and Older Adults

Matthias Hofer

Mathias Allemand

Author Note

Matthias Hofer, Institute of Mass Communication and Media Research, University of Zurich;

Mathias Allemand ( $\mathrm{PhD}$, University of Zurich) is professor at the Institute of Psychology,

University of Zurich, Switzerland.

Correspondence concerning this article should be addressed to Matthias Hofer, Institute of Mass Communication and Media Research, University of Zurich

E-mail: m.hofer@ipmz.uzh.ch

Acknowledgments: We thank Laetitia Burkhard, Annalisa Stefanelli, and Nicole Laine who conducted the experiment and handled everything concerning participant 
Dispositional and Situational Emotion Regulation in Younger and Older Adults

Younger and older adults $(N=207)$ viewed a film scene that elicits strong negative emotions and then indicated how they had regulated their emotions. Prior to stimulus presentation, dispositional emotion regulation was measured. Older adults showed higher levels of dispositional suppression compared to younger adults. Furthermore, individual differences in dispositional expressive suppression were predictive for reappraisal and suppression in the given situation. However, dispositional suppression was more strongly related to situational suppression among older adults than among younger adults. Future directions concerning the meaning of and possible implications for age differences in coping with stressful situations are discussed.

Keywords: Emotion regulation; cognitive reappraisal; expressive suppression; dispositional and situational forms; age differences 


\section{Introduction}

Emotion regulation is an important process to maintain or even improve levels of wellbeing in old age despite of losses in physical, cognitive, and social domains. Accordingly, there is a growing interest in age-related differences in both dispositional and situational emotion regulation (Kunzmann, Kupperbusch, \& Levenson, 2005; Lawton, Kleban, Rajagopal, \& Dean, 1992). However, not much is known about how individual differences in dispositional emotion regulation correspond to situational regulation strategies, and whether these associations are moderated by age. In this study we examined dispositional and situational forms of two common emotion regulation strategies - cognitive reappraisal and suppression - in younger and older adults.

\section{Emotion Regulation}

Emotion regulation is defined as "the processes by which individuals influence which emotions they have, when they have them, and how they experience and express these emotions" (Gross, 1998, p. 275). A variety of different emotion regulation strategies have been identified. However, one can roughly distinguish two kinds of emotion regulation strategies: Antecendent- and response-focused emotion regulation (Gross, 1998, 2015). Whereas the former is concerned with a manipulation of the input system (e.g., an emotional stimulus), using the latter manipulates the output system (e.g., the expression of emotion). More precisely, antecedent-focused emotion regulation includes the selection of the situation one is in, the modification of this situation, and the focus of attention paid to aspects of any given situation, or the way of thinking about a given situation (Gross, 1998). Responsefocused emotion regulation denotes the type and the strength of reaction on a behavioral or an experiential level (Gross, 1998). Cognitive reappraisal is a form of antecedent-focused emotion regulation. More precisely, it is a cognitively oriented strategy that alters the impact of an emotion by changing the way a situation is construed or by evaluating an emotional stimulus (Gross, 1998). Suppression, on the other hand, is a response-focused strategy 
directed toward inhibiting or reducing behaviors associated with emotional responses, such as facial expressions, verbal expressions, or gestures (Gross, 1998).

\section{Age Differences in Emotions Regulation}

Several theoretical perspectives suggest age differences in emotion regulation. One explanation comes from the socioemotional selectivity theory (SST, Carstensen, Isaacowitz, $\&$ Charles, 1999). This theory assumes that the perception of time left to live motivates older adults to regulate their emotions in order to maintain and maximize emotional well-being. The perception of future time may have consequences for cognitive processes such as attention, memory, and decision-making: The "positivity effect", for instance, suggests that older adults are more sensitive to positive information and less sensitive to or avoidant of negative information (Carstensen \& Mikels, 2005; Mather \& Knight, 2005, but see Murphy \& Isaacovitz, 2008). This effect may influence how older adults use emotion regulation strategies, such as avoiding situations and stimuli that possibly deteriorate their emotional well-being (Carstensen \& Mikels, 2005). For example, older adults would avoid watching media content that is likely to elicit negative emotions, such as horror movies. An additional explanation posits that accumulating life experiences from time lived provide older adults with greater expertise in emotion regulation than younger adults (Blanchard-Fields, 2007; Charles \& Luong, 2013). Learning experiences and practice in dealing with emotional situations thus might help older adults to regulate their emotions both in everyday situations and during media exposure. Other researchers have suggested that the selection and optimization of emotion regulation processes to compensate for changes in available internal resources (e.g., internal capabilities such as cognitive control) and external resources (e.g., supportive social network, action possibilities) explain age differences in emotion regulation (Urry \& Gross, 2010). Overall, these theoretical perspectives suggest that older adults place more emphasis on emotion regulation and are better at it than younger adults. However, there are also situations in which older adults are not better at regulating their emotions than 
younger adults, for instance, when older adults suffer from cognitive impairment (Scheibe \& Carstensen, 2010; Charles, 2010).

There is accumulating empirical evidence for age-related differences in a variety of emotion regulation strategies (see Charles \& Carstensen, 2010 for a detailed discussion of the literature). In general, as people age they put more emphasis on emotion regulation and get better at it (Blanchard-Fields, 2007; Carstensen, Fung, \& Charles, 2003; Gross et al., 1997). Life-long experience and practice in emotion regulation and the increased need to adapt to age-related losses in the capacity to control one's environment are often put forth as arguments for improved emotion regulation with increasing age (Charles, 2010; Scheibe \& Carstensen, 2010). However, empirical findings regarding age differences in emotion regulation are mixed: A recent study reported that the use of cognitive reappraisal was less pronounced among older adults compared to younger age groups (Nolen-Hoeksema \& Aldao, 2011). The findings of this study were based on survey data. The type of reappraisal seems to be an important factor: Older adults are less successful following experimentally induced reappraisal instructions to decrease unpleasant emotion evoked by distressing pictures compared to younger adults (Opitz, Rauch, Terry, \& Urry, 2012). However, another study showed that in everyday problem solving, older adults endorse using reappraisal to increase positive emotions more than younger adults (Blanchard-Fields, Mienaltowski, \& Baldi, 2007; John \& Gross, 2004). Reappraisal to increase positive emotions also tends to reduce subjective distress and physiological arousal while watching distressing movies more in older adults than in middle-aged or younger adults (Shiota \& Levenson, 2009). Previous work on expressive suppression has shown that younger and older adults are similarly successful at reducing outward expressions of emotion while watching a distressing film clip (Phillips, Henry, Hosie, \& Milne, 2008; Shiota \& Levenson, 2009). However, a study by John and Gross (2004) suggests that older adults use expressive suppression less frequently than younger adults do (John \& Gross, 2004). Lohani and Isaacovitz (2014) found no age 
differences in the ability to suppress emotions. Yet another study found that the use of suppression increases with age for women, but not for men (Nolen-Hoeksema \& Aldao, 2011, see also McRae, Ochsner, Mauss, Gabrieli, \& Gross, 2008). To conclude, previous individual difference studies based on survey data suggests that older adults tend to use cognitive reappraisal more frequently than younger adults do, whereas previous evidence for agerelated differences in expressive suppression is less clear (see Scheibe \& Carstensen, 2010 for a thorough review). Moreover, experimental studies using distressing pictures or video clips and manipulating emotion regulation by instructing participants show mixed results regarding the use of reappraisal (Phillips, et al., 2008; Shiota \& Levenson, 2009) and suppression (Shiota \& Levenson, 2009).

\section{Dispositional and Situational Emotion Regulation}

Emotion regulation can be conceptualized as a dispositional tendency (or trait) to regulate emotions or as a transitory regulation process (or state) that occurs in response to a specific situation that necessitates emotion regulation. Dispositional emotion regulation refers to individual differences in an enduring and cross-situational tendency to regulate emotions in a specific situation (Gross \& John, 2003). This suggests that people differ in their tendency to regulate emotions irrespective of the situational contexts. In contrast, people may differ in how they use a specific emotion regulation strategy to deal with emotions elicited by environmental stressors (e.g., a stressful movie scene) in a given situation. Hence, situational emotion regulation is a temporary change with respect to specific situations and contexts (Gross, 1998).

The two forms of emotion regulation (dispositional and situational) are interrelated at a conceptual level, as both forms involve changes in emotional responding. However, from an empirical point of view, it is unclear whether the two forms are interrelated. It is reasonable to argue that dispositional emotion regulation gives rise to the regulation of emotions in a given situation. People with higher levels in the dispositional form of an emotion regulation strategy 
should also use the situational form of this strategy more frequently and thus report having used it on a higher level. If this is correct, it can be hypothesized that the two are correlated empirically, an issue that we investigated in the current study. Because of the different foci of the two forms (i.e., cross-situational consistency versus situation specificity), however, only moderate associations can be expected between dispositional tendencies and emotion regulation in the given situation. Indeed, a recent study in younger adults reported a moderate association $(r=.24, p<.01)$ between dispositional cognitive reappraisal and reappraisal ability, defined as the ability to actually execute cognitive reappraisal successfully (McRae, Jacobs, Ray, John, \& Gross, 2012). Blalock, Kashdan, and Farmer (2016) reported even larger associations ( $\beta$ s between .29 and .54 ) associations between situational and dispositional reappraisal and suppression. These findings imply that dispositional and situational forms of cognitive reappraisal are related but not entirely overlapping constructs.

In this study, we examined whether age moderates these associations, as no previous work compared the relationships between dispositional and situational suppression and reappraisal in younger and older adults during a stressful film scene. Research from personality development would be informative to derive hypotheses. First, a large body of research demonstrates increasing levels of differential stability across the adult years in terms of maintaining rank-order stability (Roberts \& DelVecchio, 2000). This suggests that older individuals are more stable in maintaining their relative standing on personality traits over time. Although this line of research relates to longer time periods, it is possible that older adults also demonstrate higher rank-order stability across shorter time periods. Furthermore, in a study on the aforementioned positivity effect (i.e., cognitive bias toward positive and away from negative stimuli among older adults), Li, Fung, and Isaacovitz (2011) found that this effect was especially pronounced among participants with higher dispositional reappraisal. This study indicates a certain matching of dispositional emotion regulation tendencies and emotion regulation processes in a certain situation that is especially 
pronounced among older adults. According to Charles (2010), one reason for higher associations between dispositional and situational emotion regulation among older than among younger adults could be that older adults have years of experience to act according to their disposition (see also Blanchard-Fields, 2007).

\section{The Current Study}

The current study investigated dispositional and situational emotion regulation strategies and their association in younger and older adults during watching a highly stressful film scene. The first goal was to examine age differences in emotion regulation strategies. Because age differences situational emotion regulation strategies are already reported in the study by Hofer, Burkhard, and Allemand $(2015)^{1}$, the present study focuses on age differences in dispositional emotion regulation strategies. That is, we expected that older adults should report higher levels of dispositional reappraisal (H1a) and dispositional suppression (H1b).

The second goal was to examine the association between dispositional and situational emotion regulation. We expected moderate associations within the same strategy. Put differently, dispositional reappraisal should predict situational reappraisal, but not necessarily situational suppression (H2a) and vice versa (H2b).

The third goal was to examine age as a potential moderator of the relationship between dispositional and situational emotion regulation. We expected stronger associations between the two forms of emotion regulation for older adults compared to younger adults (suppression $\mathrm{H} 3 \mathrm{a}$; reappraisal $\mathrm{H} 3 \mathrm{~b})$.

\section{Method}

\section{Participants}

Participants consisted of younger and older adults $(N=207)$. Younger adults $(n=108$; $\left.n_{\text {female }}=83\right)$ ranged in age from 18 to 28 years $(M=20.9, S D=1.9)$. Older adults $(n=99$; $\left.n_{\text {female }}=50\right)$ ranged in age from 62 to 87 years $(M=72.5, S D=7.0)$. The younger participants were undergraduate communication students at a large university. They received course credit 
for participation. The sample of older participants was drawn from the local community and was recruited from a participant pool of older study participants. For the older participants we organized an event where the results of the study were presented and possible questions were answered. Most of the older participants regularly visited courses at the senior university.

\section{Procedure}

After signing the consent form, participants were asked to respond to questionnaires asking about their current emotions, their dispositional emotion regulation, and demographic information (baseline assessment: T1). Then, participants individually sat alone in a darkened room front of a big TV screen and were told that they would be viewing a movie until a specific scene and rating their emotional reactions and answering questions after this scene. They were given headphones. After 12 minutes and 52 seconds, we stopped the film and asked participants to indicate their current emotions as well as their use of situational emotional regulation with respect to the highly emotional scene in the movie (follow-up assessment after the film stimulus: T2). In order to ensure that participants answered the items on situational emotion regulation with regard to the specific situation (i.e., the regulation of emotions while watching the movie scene), we presented four stills depicting the most emotional scenes to the participants and asked them to think about how they regulated their emotions during the film.

The experiment took place in single sessions and each participant sat alone in the room while watching the film scene and answering the questionnaire. A research assistant was always available in case participants needed assistance or had questions.

\section{Film Stimulus and Emotion Manipulation Check}

To elicit an increase in anger and sadness and a decrease in happiness, respectively, we used the first five minutes of a shortened version of the first part of the movie "Dancer in the Dark” (USA, 2000, directed by Lars von Trier, rated PG-13). The movie features the Czech immigrant Selma Ježková. Selma has moved to the United States with her son Gene and lives 
a life in poverty in a mobile trailer on the property of town police officer Bill Houston. She suffers from a hereditary degenerative disease diminishing her eyesight gradually and inexorably. Also Gene suffers from this disease. In order to pay an operation that will prevent her son from suffering the same fate, she saves up every penny she earns. One day, Bill steels Selma's life savings. When Selma finds out about the theft, she confronts Bill. The situation gets out of hand. Finally, Bill begs Selma to kill him, because he cannot stand his life anymore. Therefore Selma shoots him. The scene ends with a focus on Selma sitting next to Bill and crying. It is important to note that no participant had seen the movie before or was familiar with the plot.

\section{Measures}

Emotions. We used adjectives to measure anger (furious, angry, irate), sadness (downhearted, despondent, sad), and happiness (balanced, feel good, happy) at baseline (T1) and after the film stimulus (T2). Participants indicated how well the adjectives describe their current emotions using a Likert-type scale ranging from 1 (not at all) to 5 (very strong). The alpha reliabilities (T1 and T2) were: .92, .89 (anger), .83, .79 (sadness), and .78, .85 (happiness).

Dispositional emotion regulation. The 10-item Emotion Regulation Questionnaire (ERQ; Gross \& John, 2003) was used to measure individual differences in dispositional cognitive reappraisal and expressive suppression at T1 (see procedure above). Six items assess people's use of reappraisal (e.g., "When I want to feel more positive emotion (such as joy or amusement) I change what I'm thinking about"; "I control my emotions by changing the way I think about the situation I'm in"). Four items assess people's use of suppression (e.g., "I keep my emotions to myself"; "When I am feeling negative emotions, I make sure not to express them”). Responses were made on a Likert-type scale ranging from 1 (strongly agree) to 5 (strongly agree). The alpha reliabilities were: .70 (reappraisal) and .74 (suppression). 
Situational emotion regulation. Two short scales were used to measure how participants perceive their emotion regulation strategies with respect to the highly emotional film scene at T2 (see procedure above). We rephrased items for dispositional emotion regulation (see also Blalock et al., 2016; Nezlek \& Kuppens, 2008). The items to assess reappraisal (4 items, e.g., "I tried to think differently about the scene in order to have less intense emotions") and suppression (4 items, e.g., "I tried to suppress my feelings by not showing them.") were developed in accordance with Gross and John (2003), but with a focus on situational emotion regulation. Responses were made on a Likert-type scale ranging from 1 (not at all) to 5 (very strong). The alpha reliabilities were: .70 (reappraisal) and .88 (suppression).

\section{Results}

\section{Emotion Manipulation Check}

We first examined whether the film stimulus was successful in eliciting negative emotions of anger and sadness and decreased happiness. We performed a series of repeatedmeasures ANOVA's for each of the three emotions (anger, sadness, happiness) as the withinsubjects variable and age group (younger, older) as the between-subjects factor and controlled for potential gender effects. For anger, we found a significant increase from T1 $(M=1.28, S E$ $=0.05)$ to $\mathrm{T} 2(M=3.37, S E=0.09), F(1,199)=105.50, p=.00$, partial $\eta^{2}=.35$. These changes were independent of the age group $\left(F(1,199)=.30, p=.59\right.$, partial $\left.\eta^{2}=.00\right)$. That is, younger adults reported slightly higher values of anger at T1 $(M=1.37, S E=0.84)$ than older adults $\left(M=1.20, S E=0.60, F(1,199)=2.83, p=.10\right.$, partial $\left.\eta^{2}=.01\right)$. At T2, younger $(M=3.43, S E=1.15)$ and older adults did not differ in terms of anger $(M=3.26, S E=1.31$; $F(1,199)=.63, p=.42$, partial $\left.\eta^{2}=.00\right)$

Sadness also increased from T1 $(M=1.53, S E=0.06)$ to T2 $(M=3.25, S E=0.08), F(1$, $198)=70.12, p=.00$, partial $\eta^{2}=.26$. The results indicated that changes in sadness were independent of the age group, $F(1,199)=.13, p=.72$, partial $\eta^{2}=.00$. That is, in terms of 
sadness, younger adults reported higher values at $\mathrm{T} 1(M=1.66, S E=0.89)$ than older adults $\left(M=1.39, S E=0.70 ; F(1,199)=5.89, p=.02\right.$, partial $\left.\eta^{2}=.03\right)$. We found the same pattern at T2: Younger adults were sadder $(M=3.41, S E=1.06)$ than older adults $(M=3.08, S E=$ $1.22 ; F(1,199)=4.04, p=.05$, partial $\left.\eta^{2}=.02\right)$.

For happiness, we found a decrease from T1 $(M=4.00, S E=0.06)$ to T2 $(M=1.39, S E$ $=0.04), F(1,200)=390.23, p=.00$, partial $\eta^{2}=.66$. The results also indicated an interaction between emotional change and the age group, $F(1,200)=13.77, p=.00$, partial $\eta^{2}=.06$. Younger adults reported lower levels of happiness at $\mathrm{T} 1(M=3.84, S E=0.08)$ compared to older adults $\left(M=4.16, S E=0.08 ; F(1,199)=7.70, p=.01\right.$, partial $\left.\eta^{2}=.04\right)$, whereas they had slightly higher levels at T2 $(M=1.46, S E=0.06)$ compared to older adults $(M=1.33, S E$ $=0.06$ ). Overall, the results suggest that the film was successful at eliciting negative emotions and to decrease positive emotions.

\section{Age Differences in Emotion Regulation}

Table 1 contains descriptive statistics and zero-order correlations. On average, participants reported significant higher levels in dispositional cognitive reappraisal as compared to expressive suppression, $t(205)=8.94, p<.01, d=.62$. The means of the two situational emotion regulation strategies did not differ from each other.

$\mathrm{H} 1 \mathrm{a}$ and $\mathrm{H} 1 \mathrm{~b}$ predicted age difference in dispositional suppression and dispositional reappraisal with higher levels among older adults than among younger adults. To examine these age differences, we first estimated a multivariate analysis of variance (MANOVA) with age group as independent variable and the regulation strategies as dependent variables. This analysis revealed a strong main effect of age: Wilks' $\Lambda=0.89, F(4,203)=13.25$, partial $\eta^{2}$ $=.12, p<.001$. Next, we estimated a series of one-way ANOVA's and controlled for potential gender effects. Table 2 contains the estimates for the emotion regulation strategies by age groups and $95 \%$ confidence intervals. While younger and older adults did not differ in 
dispositional cognitive reappraisal, $F(1,205)=.13, p<.72$, partial $\eta^{2}=.00$, older adults had higher levels of dispositional suppression than younger adults, $F(1,205)=17.31, p=.00$, partial $\eta^{2}=.08$. We also tested for possible age by gender interactions for each of the four emotion regulation strategies. None of the age by gender interactions was significant. Overall, we evidenced age differences in dispositional suppression strategies, but not for dispositional cognitive reappraisal. Thus, our data are in support for H1b. H1a could not be supported.

\section{Associations between the Two Forms of Emotion Regulation}

H2a predicted that dispositional reappraisal should predict situational reappraisal, but not necessarily situational suppression. $\mathrm{H} 2 \mathrm{~b}$ predicted that dispositional suppression should predict situational suppression, but not necessarily situational reappraisal. The results in Table 1 suggest that cognitive reappraisal and expressive suppression were interrelated both at the dispositional and the situational level. More importantly, dispositional reappraisal at T1 was prospectively related to situational reappraisal at $\mathrm{T} 2(r=.17, p=.01)$. This correlation can be considered as rather small (Hemphill, 2003). We found a large correlation between dispositional expressive suppression at T1 and situational suppression at T2 $(r=.48, p=.01)$. Hence, those participants with higher levels in dispositional reappraisal and suppression, respectively, also tended to report higher levels of reappraisal and suppression, respectively, after viewing the film scene. A test of the difference between the two independent correlation coefficients $(.17 ; .48)$ indicated that the strength of relationships were statistically different, $z$ $=3.55, p=.00$. Further, the results in Table 1 indicated that dispositional suppression was prospectively related to situational reappraisal.

\section{Multivariate and Moderator Tests}

To test the multivariate associations between dispositional and situational regulation strategies and the examine the moderating role of age, as predicted in $\mathrm{H} 3 \mathrm{a}$ and $\mathrm{H} 3 \mathrm{~b}$, we estimated two regression models using the PROCESS computational procedures (Model 1) for probing interactions by Hayes (2013). The PROCESS macro produces the usual 
regression output and estimates of the effect of the focal predictor variables at values of the moderator variable $(0=$ younger adults, $1=$ older adults $)$. We estimated ordinary least squares (OLS) regression models with each of the two situational emotion regulation strategies as outcome variables, the respective form of dispositional emotion regulation as the focal predictor and age group as moderator. Gender was included as covariate in all analyses. Moreover, in the model for situational suppression (see Table 3) we controlled for the effect of dispositional reappraisal, while we controlled for dispositional reappraisal in the model for situational suppression (see Table 4).

Results show that age predicted situational suppression was predicted by age $(b=0.33$, $S E=0.12, t(198)=2.66, p=.01)$ and dispositional suppression $(b=0.31, S E=0.10, t(198)=$ $3.28, p=.00)$. In addition, age moderated the link between dispositional and situational suppression. This association was less pronounced in younger adults $(b=0.32, S E=0.10$, $t(198)=3.28, p=.00,95 \% \mathrm{CI}=0.13 ; 0.51)$ compared to older adults $(b=0.66, S E=0.11$, $t(198)=6.22, p=.00,95 \% \mathrm{CI}=0.45 ; 0.86)$. These results indicate that expressive suppression as a response to the film stimulus is more strongly related to its dispositional form in older adults compared to younger adults. Thus, we found support for H3a.

As one can see in Table 4, situational reappraisal was positively predicted by age group $(b=0.36, S E=0.14, t(199)=2.55, p=.01)$. That is, older adults showed higher tendency to use reappraisal as a strategy to regulate their emotions during watching the film scene (see also Table 2). Rather surprisingly, dispositional suppression and not dispositional reappraisal predicted situational reappraisal. In addition, age did not moderate the association between dispositional and situational reappraisal. Thus, the results in Table 4 are not in support of H3b. The results demonstrated that age did not moderate the relationship between dispositional cognitive reappraisal and situational reappraisal as well as between dispositional suppression and situational reappraisal. 
One could argue that including changes in momentary emotional experiences as covariates would be an even stronger test of our theoretical predictions. Therefore, in additional models we included change scores in anger, sadness, and happiness as covariates. The inclusion of change scores did not significantly alter the results. Results of these analyses can be found in the Appendix A (Table 5 and Table 6).

\section{Discussion}

The current study investigated age differences in emotion regulation during a highly stressful film scene film. With respect to our first hypotheses (H1a and H1b), older adults showed higher levels in dispositional and situational suppression. In general, this finding fits with past theoretical and empirical work demonstrating that, as people age, they put more emphasis on emotion regulation (Blanchard-Fields, 2007; Charles \& Carstensen, 2010; Carstensen et al., 2003; Gross et al., 1997). However, we did not find age differences in dispositional reappraisal. This could be due to our sample of younger adults which consisted of students. It could be that students are generally more used to use cognitive strategies (for instance while preparing for or writing exams). Future studies should therefore work with samples from a broader population.

The main objective of the present study relates to the link between dispositional and situational emotion regulation. In support of our expectation, results indicate that individual differences in dispositional emotion regulation were predictive for the regulation of negative emotions in response to the film scene. Overall, these results are in line with McRae et al.'s (2012) findings and contribute to other work demonstrating that people with higher levels on a personality trait experienced greater increases in the state corresponding to this trait (e.g., Harrigan, Lucic, \& Rosenthal, 1991). Moreover, the current results showed that the link between dispositional suppression and situational suppression was stronger than the link between dispositional cognitive reappraisal and situational reappraisal. Put differently, high scores in dispositional suppression were more predictive for suppression in the given situation 
than high levels of dispositional reappraisal was for situational reappraisal. Moreover, looking at the multivariate results, we found no association between dispositional and situational reappraisal. This could be due to the stimulus used in the present study. The last minutes of the film scene depicts a highly distressing situation (i.e., Selma killing Bill). As a result, in terms of situational regulation which can be conceived of as a "trait-relevant" state (Fleeson, 2001, p. 1012), there is not much opportunity for "reappraisers" in this situation to act upon their disposition. Future research could test this by experimentally manipulating suppression or reappraisal (see for example Shiota \& Levenson, 2009).

A finding worth noting is the positive association between dispositional suppression and situational reappraisal. One explanation for this finding could be found in the fact that situational suppression and situational reappraisal are not orthogonal concepts; both are strategies to regulate one's emotions. In fact, our data shows that they are highly correlated ( $r$ $=.41, p=.01$, see Table 1). Although speculative, one could argue that high "suppressors" use any form of emotion regulation, as long as they do not have to express their emotions. In addition, one has to keep in mind that in Gross's model, emotion regulation is conceived of as a circular process (Gross, 2015). That is, once an emotion is elicited by some stimulus, a person can choose to suppress it. However, research shows that emotions do not disappear by suppressing them (Gross, 1998). Thus, the process starts anew and reappraisal can come into play and so forth. It may be the case that the situation we created (i.e., the very strongly emotional movie scene) has lead "suppressors" to use any means (i.e., both suppression and reappraisal) in order not to experience negative emotions. Future studies could test this assumption by more closely examining associations between dispositional suppression and antecedent- and response-focused situational emotion regulation strategies in a given situation with a higher temporal resolution of the assessment of situational regulation strategies to account for dynamics in emotion regulation over time. 
Finally, we examined the moderating role of age. Our results suggest that age moderates the link between the two forms of suppression. Dispositional suppression was more strongly related to situational suppression in older adults as compared to younger adults. This result indicates that individual differences in dispositional emotion regulation are more important for the prediction of older adults' emotion regulation in the situation than younger adults' situational emotion regulation, at least for expressive suppression. This finding is in line with work demonstrating age-related differences in variability with respect to self-perceptions in different situations and at different times (McReynolds, Altrocchi \& House, 2000). For instance, McReynolds and colleagues found that older people tend to see themselves as less variable in their cognitions, feelings, and behaviors than younger persons do. It is then possible that the behavioral variability in a given situation such as the present media exposure situation study is more restricted for older adults than for younger adults. As a consequence, their situational suppression might be related more strongly to the corresponding trait.

Our work is not without limitations: First, we only focused on two commonly used emotion regulation strategies. Future research may include other emotion regulation strategies, as previous research evidenced age differences in a variety of emotion regulation strategies, such as distraction (Knight et al., 2007) or rumination (Nolen-Hoeksema \& Aldao, 2011). Similarly, future research may include dispositions such as the Big Five traits as control or moderating variables in addition to dispositional aspects of emotion regulation, because the experience and regulation of emotions are directly related to personality traits (Gross \& John, 2003). Second, self-reports of emotion regulation may not always be valid and thus can be supplemented by behavioral measures. Another limitation was the use of one single film stimulus. It is possible that age differences in emotion regulation are partly due to the chosen stimulus. Future research may replicate the current findings using other stimuli. However, it is important to note that the film elicited negative emotions equally well in younger and older adults in our study. In addition, we did not assess the regulation success. 
That is, we did not examine whether participants regarded themselves as successful in dealing with the negative affect or whether the experienced affect was downregulated at all (see Shiota \& Levenson, 2009). Next, we cannot rule out that the differences in emotion regulation are due to cohort rather than developmental effects. Therefore, future research should apply longitudinal designs that can account for such effects. Finally, it should be mentioned that we worked with a convenience sample and that the gender distributions were uneven in the two age groups.

\section{Conclusion and Implications}

In conclusion, this study provides a valuable insight into the relationship between dispositional and situational emotion regulation strategies in younger and older adults during watching an emotionally distressing film clip. In particular, we found evidence that individual differences in dispositional emotion regulation predict the regulation of negative emotions in a given situation that elicits negative emotions, though slightly stronger for older adults than younger adults in the case of expressive suppression. As discussed above, older adults seem to be more stable in their trait-relevant behavior due to their life-long experience in dealing with emotional situations than younger adults. However, the fact that we did not find this association between dispositional and cognitive reappraisal points to the fact that-aside from personality as a determinant of behavior-the situation itself can play a major role. This finding also implies that in certain cases, suppression might be more adaptive than reappraisal, although in the long-run suppressing one's emotions has rather negative effects (John \& Gross, 2004). The current results may inform future research on the benefits of different forms of emotion regulation strategies in different situations in old age. The results clearly emphasize the importance of taking both forms of emotion regulation into account when studying age differences in emotion regulation. 


\section{Notes}

${ }^{1}$ It should be mentioned that we used the same sample and the same dataset in Hofer et al., (2015) and in this study. However, the main goal of the study by Hofer et al. (2015) was to examine measurement invariance of situational emotion regulation strategies. In addition to the psychometric issues we reported age differences in the latent means of situational (but not dispositional) emotion regulation strategies. The present study builds on these results, but its main goal is to examine how dispositional and situational regulation strategies are associated and whether these associations are moderated by age. 


\section{References}

Blalock, D. V., Kashdan, T. B., \& Farmer, A. S. (2016). Trait and Daily Emotion Regulation in Social Anxiety Disorder. Cognitive Therapy and Research, 40, 416-425. doi:10.1007/s10608-015-9739-8

Blanchard-Fields, F. (2007). Everyday problem solving and emotion: An adult developmental perspective. Current Directions in Psychological Science, 16, 26-31. doi: 10.1111/j.1467-8721.2007.00469.x

Blanchard-Fields, F., Mienaltowski, A., \& Baldi, R. (2007). Age differences in everyday problem-solving effectiveness: Older adults select more effective strategies for interpersonal problems. Journals of Gerontology: Psychological Sciences, 62B, 61-64.

Carstensen, L. L., Fung, H. H., \& Charles, S. T. (2003). Socioemotional selectivity theory and the regulation of emotion in the second half of life. Motivation and Emotion, 27(2), 103-123. doi:10.1023/A:1024569803230

Carstensen, L. L., Isaacowitz, D. M., \& Charles, S. T. (1999). Taking time seriously: A theory of socioemotional selectivity. American Psychologist, 54, 165-181.

Carstensen, L. L., \& Mikels, J. A. (2005). At the intersection of emotion and cognition. Aging and the positivity effect. Current Directions in Psychological Science, 14(3), 117-121. doi:10.1111/j.0963-7214.2005.00348.x

Charles, S. T. (2010). Strength and vulnerability integration: A model of emotional well-being across adulthood. Psychological Bulletin, 136, 1068-1091. https://doi.org/10.1037/a0021232

Charles, S. T., \& Carstensen, L. L. (2010). Social and emotional aging. Annual Review of Psychology, 61, 383-409. doi:10.1146/annurev.psych.093008.100448

Charles, S. T., \& Luong, G. (2013). Emotional experience across adulthood: The theoretical model of strength and vulnerability integration. Current Directions in Psychological Science, 22, 443-448. doi:10.1177/0963721413497013 
Fleeson, W. (2001). Toward a structure- and process-integrated view of personality: Traits as density distributions of states. Journal of Personality and Social Psychology, 80, 10111027. doi:10.1037/0022-3514.80.6.1011

Gross, J. J. (1998). The emerging field of emotion regulation: An integrative review. Review of General Psychology, 2(3), 271-299. doi:10.1037/1089-2680.2.3.271

Gross, J. J. (2015). Emotion regulation: Current status and future prospects. Psychological Inquiry, 26, 1-26. doi:10.1080/1047840X.2014.940781

Gross, J. J., Carstensen, L. L., Pasupathi, M., Tsai, J., Götestam Skorpen, C., \& Hsu, A. Y. C. (1997). Emotion and aging: Experience, expression, and control. Psychology and Aging, 12(4), 590-599. doi:10.1037/0882-7974.12.4.590

Gross, J. J., \& John, O. P. (2003). Individual differences in two emotion regulation processes: Implications for affect, relationships, and well-being. Journal of Personality and Social Psychology, 85(2), 348-362. doi:10.1037/0022-3514.85.2.348

Harrigan, J. A., Lucic, K. S., \& Rosenthal, R. (1991). Retelling anxious events: Effects on trait and state anxiety. Personality and Individual Differences, 12, 917-927.

Hayes, A. F. (2013). Introduction to mediation, moderation, and conditional process analysis: A regression-based approach. New York, NY: Guilford Press.

Hemphill, J. F. (2003). Interpreting the magnitudes of correlation coefficients. American Psychologist, 58, 78-79. doi:10.1037/0003-066X.58.1.78

Hofer, M., Burkhard, L., \& Allemand, M. (2015). Age differences in emotion regulation during a distressing film scene. Journal of Media Psychology: Theories, Methods, and Applications, 27, 1-6. https://doi.org/10.1027/1864-1105/a000134

John, O. P., \& Gross, J. J. (2004). Healthy and unhealthy emotion regulation: Personality processes, individual differences, and life span development. Journal of Personality, 72, 1301-1334. doi:10.1111/j.1467-6494.2004.00298.x 
Knight, M., Seymour, T. L., Gaunt, J. T., Baker, C., Nesmith, K., \& Mather, M. (2007). Aging and goal-directed emotional attention: Distraction reverses emotional biases. Emotion, 7, 705-714. doi:10.1037/1528-3542.7.4.705

Kunzmann, U., Kupperbusch, C. S., \& Levenson, R. W. (2005). Behavioral inhibition and amplification during emotional arousal: A comparison of two age groups. Psychology and Aging, 20, 144-158. doi:10.1037/0882-7974.20.1.144

Lawton, P. M., Kleban, M. H., Rajagopal, D., \& Dean, J. (1992). Dimensions of affective experience in three age groups. Psychology and Aging, 7, 171-184. doi:10.1037//08827974.7.2.171

Li, T., Fung, H. H., \& Isaacowitz, D. M. (2011). The role of dispositional reappraisal in the age-related positivity effect. The journals of gerontology. Series B, Psychological sciences and social sciences, 66, 56-60. doi:10.1093/geronb/gbq074

Lohani, M., \& Isaacowitz, D. M. (2014). Age differences in managing response to sadness elicitors using attentional deployment, positive reappraisal and suppression. Cognition \& Emotion, 28, 678-697. doi:10.1080/02699931.2013.853648

Mather, M., \& Knight, M. (2005). Goal-directed memory: The role of cognitive control in older adults' emotional memory. Psychology and Aging, 20, 554-570. doi:10.1037/0882-7974.20.4.554

McRae, K., Jacobs, S. E., Ray, R. D., John, O. P., \& Gross, J. J. (2012). Individual differences in reappraisal ability: Links to reappraisal frequency, well-being, and cognitive control. Journal of Research in Personality, 46, 2-7. doi:10.1016/j.jrp.2011.10.003

McRae, K., Ochsner, K. N., Mauss, I. B., Gabrieli, J. J. D., \& Gross, J. J. (2008). Gender Differences in Emotion Regulation: An fMRI Study of Cognitive Reappraisal. Group Processes \& Intergroup Relations, 11, 143-162.

https://doi.org/10.1177/1368430207088035 
McReynolds, P., Altrocchi, J., \& House, C. (2000). Self-pluralism: Assessment and relations to adjustment, life changes, and age. Journal of Personality, 68, 347-381. doi:10.1111/1467-6494.00100

Murphy, N. A., \& Isaacowitz, D. M. (2008). Preferences for emotional information in older and younger adults: a meta-analysis of memory and attention tasks. Psychology and Aging, 23, 263-286. doi:10.1037/0882-7974.23.2.263

Nezlek, J. B., \& Kuppens, P. (2008). Regulating positive and negative emotions in daily life. Journal of Personality, 76, 561-580. doi:10.1111/j.1467-6494.2008.00496.x

Nolen-Hoeksema, S., \& Aldao, A. (2011). Gender and age differences in emotion regulation strategies and their relationship to depressive symptoms. Personality and Individual Differences, 51, 704-708. doi:10.1016/j.paid.2011.06.012

Opitz, P. C., Rauch, L. C., Terry, D. P., \& Urry, H. L. (2012). Prefrontal mediation of age differences in cognitive reappraisal. Neurobiology of Aging, 33, 645-655. doi:10.1016/j.neurobiolaging.2010.06.004

Phillips, L. H., Henry, J. D., Hosie, J. A., Milne, A. B. (2008). Effective regulation of the experience and expression of negative affect in old age. Journals of Gerontology: Psychological Sciences, 36B, 138-145.

Roberts, B. W., \& DelVecchio, W. F. (2000). The rank-order consistency of personality traits from childhood to old age: A quantitative review of longitudinal studies. Psychological Bulletin, 126, 3-25. doi:10.1037/0033-2909.126.1.3

Scheibe, S., \& Carstensen, L. L. (2010). Emotional aging: recent findings and future trends. The journals of gerontology. Series B, Psychological sciences and social sciences, $65 B$, 135-144. doi:10.1093/geronb/gbp132

Shiota, M. N., \& Levenson, R. W. (2009). Effects of aging on experimentally instructed detached reappraisal, positive reappraisal, and emotional behavior suppression. Psychology and Aging, 24, 890-900. doi:10.1037/a0017896 . 
Urry, H. L., \& Gross, J. J. (2010). Emotion regulation in older age. Current Directions in Psychological Science, 19, 352-357. doi:10.1177/0963721410388395 . 
Table 1

Descriptive Statistics and Correlations Among the Emotion Regulation Strategies

\begin{tabular}{lcccccc}
\hline & 1 & 2 & 3 & 4 & 5 & 6 \\
\hline 1. Age & - & & & & & \\
2. Gender & & & & & & \\
3. Dispositional cognitive reappraisal & .03 & .08 & - & & & \\
4. Dispositional expressive suppression & $.36^{* *}$ & $-.28^{* *}$ & $.20^{* *}$ & - & & \\
5. Situational cognitive reappraisal & $.23^{* *}$ & -.07 & $.17^{*}$ & $.25^{* *}$ & - & \\
6. Situational expressive suppression & $.33^{* *}$ & $-.14^{*}$ & .07 & $.48^{* *}$ & $.41^{* *}$ & - \\
$M$ & 44.08 & - & 3.28 & 2.70 & 2.10 & 2.07 \\
SD & 26.18 & - & 0.69 & 0.87 & 0.96 & 0.94
\end{tabular}

Note. $N=207$; dispositional emotion regulation strategies were assessed at baseline (T1), whereas situational strategies were assessed at follow-up (T2); the potential range of the emotion regulation strategies were $1-5 ;{ }^{\mathrm{a}} 0=$ male, $1=$ female; ${ }^{1}$ The negative correlation between age and gender is due to different gender distributions among older $\left(n_{\text {female }}=50 ; n_{\text {male }}\right.$ $=47)$ and younger $\left(n_{\text {female }}=83 ; n_{\text {male }}=25\right)$ participants; ${ }^{*} p<.05, * * p<.01$. 
Table 2

Emotion Regulation Strategies by Age

\begin{tabular}{lccccc}
\hline & \multicolumn{3}{c}{ Younger adults } & & \multicolumn{2}{c}{ Older adults } \\
\cline { 2 - 6 } & $M$ & $S E$ & $95 \%$ CI & $M$ & $S E$ \\
\hline Dispositional cognitive reappraisal & $3.28 \mathrm{a}$ & 0.07 & $3.15 ; 3.43$ & $3.35 \mathrm{a}$ & 0.07 \\
Dispositional expressive suppression & $2.46_{\mathrm{a}}$ & 0.08 & $2.31 ; 2.62$ & $2.95 \mathrm{~b}$ & $3.21 ; 3.48$ \\
Situational cognitive reappraisal $^{1}$ & $1.89 \mathrm{a}$ & 0.09 & $1.71 ; 2.08$ & $2.35 \mathrm{~b}$ & 0.08 \\
Situational expressive suppression $^{1}$ & $1.81_{\mathrm{a}}$ & 0.09 & $1.66 ; 1.96$ & $2.37 \mathrm{~b}$ & 0.09
\end{tabular}

Note. $n=108$ younger adults, $n=99$ older adults; the estimates are adjusted for gender effects; $95 \%$ CI: bias-corrected and accelerated 95\%

confidence intervals based on 5000 bootstrap samples; different subscripts (a and/or b) indicate significant differences between younger and older adults at $p<.05$ (for more details, see the results section). ${ }^{1}$ Note that age differences in situational emotion regulation strategies are reported in

Hofer et al. (2015). To avoid confusion it has to be mentioned that the differences in Hofer et al. (2015) are differences in latent means. 


\section{Table 3}

Moderated Regression Analysis of Situational Suppression

\begin{tabular}{lcccccc}
\hline Predictors & $B$ & $S E$ & $\beta$ & $p$ & $L L C I$ & $U L C I$ \\
\hline Intercept & & & & & & \\
Sex $(0=$ male) & 1.83 & 0.12 & & .00 & 1.58 & 2.07 \\
Age group (0 = younger adults) & 0.07 & 0.13 & .04 & .68 & -0.18 & 0.32 \\
Dispositional suppression (mean centered) & 0.33 & 0.21 & .31 & .01 & 0.09 & 0.57 \\
Age group*dispositional suppression (mean centered) & 0.32 & 0.10 & .29 & .00 & 0.13 & 0.51 \\
Dispositional reappraisal (mean centered) & 0.34 & 0.14 & .57 & .02 & 0.06 & 0.61 \\
\multicolumn{1}{c}{$R^{2}$} & -0.04 & 0.09 & -.03 & .68 & -0.20 & 0.13
\end{tabular}

Simple effects of dispositional suppression for younger and older adults

\begin{tabular}{lllllll}
\hline Age group & & $B$ & $S E$ & $p$ & $L L C I$ & $U L C I$ \\
\hline & Younger adults & 0.32 & 0.10 & .00 & 0.13 & 0.51 \\
& Older adults & 0.66 & 0.11 & .00 & 0.45 & 0.86 \\
\hline
\end{tabular}


Table 4.

Moderated Regression Analysis of Situational Reappraisal

\begin{tabular}{lcccccc}
\hline Predictors & $B$ & $S E$ & $\beta$ & $p$ & $L L C I$ & $U L C I$ \\
\hline Intercept & 1.92 & 0.14 & & .00 & 1.64 & 2.19 \\
Sex $(0=$ male) & 0.04 & 0.15 & .02 & .78 & -0.25 & 0.33 \\
Age group (0 = younger adults) & & & & & \\
Dispositional reappraisal (mean centered) & 0.36 & 0.14 & .19 & .01 & 0.08 & 0.64 \\
Age group*dispositional reappraisal (mean centered) & 0.15 & 0.13 & .10 & .26 & -0.11 & 0.40 \\
Dispositional suppression (mean centered) & 0.11 & 0.19 & .17 & .58 & -0.27 & 0.49 \\
\multicolumn{1}{c}{$R^{2}$} & 0.20 & 0.08 & .05 & .02 & 0.03 & 0.36
\end{tabular}

Simple effects of dispositional reappraisal for younger and older adults

\begin{tabular}{lllllll}
\hline Age group & & $B$ & $S E$ & $p$ & LLCI & ULCI \\
\hline & Younger adults & 0.15 & 0.13 & .26 & -0.11 & 0.40 \\
& Older adults & 0.25 & 0.15 & .09 & -0.04 & 0.54 \\
\hline
\end{tabular}




\section{APPENDIX}

Table 5.

Moderated Regression Analysis of Situational Suppression

\begin{tabular}{lcccccc}
\hline Predictors & $B$ & $S E$ & $\beta$ & $p$ & $L L C I$ & $U L C I$ \\
\hline intercept & 1.84 & 0.13 & & .00 & 1.59 & 2.10 \\
Sex (0 = male) & 0.04 & 0.13 & .02 & .78 & -0.22 & 0.29 \\
Age group (0 = younger adults) & 0.34 & 0.13 & .18 & .01 & 0.08 & 0.60 \\
Dispositional suppression (mean & & & .31 & .00 & & \\
centered) & 0.33 & 0.10 & & & 0.13 & 0.53 \\
Age group*dispositional suppression & 0.29 & 0.14 & .50 & .04 & 0.01 & 0.57 \\
Dispositional reappraisal (mean centered) & -0.04 & 0.09 & -.03 & .62 & -0.22 & 0.13 \\
Change score happiness (mean centered) & 0.06 & 0.06 & .06 & .37 & -0.07 & 0.18 \\
Change score sadness (mean centered) & 0.05 & 0.05 & .07 & .37 & -0.06 & 0.15 \\
Change score anger (mean centered) & 0.02 & 0.05 & .04 & .63 & -0.07 & 0.12 \\
\multicolumn{1}{c}{$R^{2}$} & & & 0.26 & & & \\
\hline
\end{tabular}

Simple effects of dispositional suppression for younger and older adults

\begin{tabular}{llccccc}
\hline Age group & & $B$ & $S E$ & $p$ & $L L C I$ & $U L C I$ \\
\hline & Younger adults & 0.33 & 0.10 & .00 & 0.13 & 0.53 \\
& Older adults & 0.62 & 0.11 & .00 & 0.40 & 0.84 \\
\hline
\end{tabular}


Table 6.

Moderated Regression Analysis of Situational Reappraisal

\begin{tabular}{lcccccc}
\hline Predictors & $B$ & $S E$ & $\beta$ & $p$ & $L L C I$ & $U L C I$ \\
\hline intercept & 1.95 & 0.14 & & .00 & 1.66 & 2.23 \\
Sex (0 = male) & 0.02 & 0.15 & .01 & .89 & -0.27 & 0.31 \\
Age group (0 = younger adults) & 0.33 & 0.15 & .17 & .03 & 0.04 & 0.62 \\
Dispositional reappraisal (mean centered) & 0.16 & 0.13 & .11 & .23 & -0.10 & 0.41 \\
Age group*dispositional reappraisal & 0.04 & 0.20 & .02 & .84 & -0.35 & 0.43 \\
Dispositional suppression (mean & & & .22 & .01 & & \\
centered) & 0.25 & 0.09 & & & 0.07 & 0.42 \\
Change score happiness (mean centered) & 0.01 & 0.07 & .01 & .91 & -0.13 & 0.15 \\
Change score sadness (mean centered) & 0.02 & 0.06 & .03 & .73 & -0.10 & 0.14 \\
Change score anger (mean centered) & 0.09 & 0.06 & .14 & .10 & -0.02 & 0.20 \\
$\quad R^{2}$ & & & 0.13 & & &
\end{tabular}

Simple effects of dispositional reappraisal for younger and older adults

\begin{tabular}{llccccc}
\hline Age group & & $B$ & $S E$ & $p$ & $L L C I$ & $U L C I$ \\
\hline & Younger adults & 0.16 & 0.13 & .23 & -0.10 & 0.41 \\
& Older adults & 0.20 & 0.15 & .19 & -0.10 & 0.49 \\
\hline
\end{tabular}

